\title{
Gated SPECT myocardial perfusion imaging quality assurance in current and future practice
}

\author{
Kenneth J. Nichols, PhD, ${ }^{\mathrm{a}}$ and Andrew Van Tosh, $\mathrm{MD}^{\mathrm{b}}$ \\ a Division of Nuclear Medicine and Molecular Imaging, Northwell Health, New Hyde Park, NY \\ b Research Department, St. Francis Hospital, Roslyn, NY
}

Received Dec 2, 2016; accepted Dec 2, 2016

doi:10.1007/s12350-016-0752-4

\section{See related article, pp. 534-542}

Conducting a valid medical imaging study requires diligent quality assurance (QA) of imaging equipment, attention to all patient-specific details during data acquisition, assessment of data integrity of acquired data once the study is complete, and careful computer processing of image data. ${ }^{1}$ In the current issue of the Journal of Nuclear Cardiology, Malek et $\mathrm{al}^{2}$ review some of the important elements of QA procedures required to produce reliable computations of left ventricular (LV) perfusion and function parameters from gated SPECT myocardial perfusion imaging (MPI) data for the Cedars-Sinai QGS program, ${ }^{3}$ which is a commonly used commercially available MPI analysis software package. While other algorithms operate on somewhat different principles and LV modeling assumptions, ${ }^{4,5}$ all MPI software packages have in common the automated identification of the LV outflow tract valve plane, endocardial and epicardial borders. As the authors remind us, failure to ensure the appropriate locations of LV limits can compromise the validity of summed stress scores, summed rest scores, ejection fractions, LV volumes, LV mass, lung:heart count ratios, and transient ischemic dilatation, among other parameters.

Reprint requests: Kenneth J. Nichols, Division of Nuclear Medicine and Molecular Imaging, Northwell Health, 270-05 76th Avenue, New Hyde Park, NY 11040; knichols@northwell.edu

FundingKenneth Nichols participates in royalties from Syntermed, Inc., in relation to some of the algorithms discussed in this manuscript. Andrew Van Tosh serves as a consultant to Astellas Pharmaceuticals, Inc.

J Nucl Cardiol 2017;24:543-5.

$1071-3581 / \$ 34.00$

Copyright (C) 2016 American Society of Nuclear Cardiology.
Additional parameters that can be compromised by sub-optimal LV valve plane placement include phase maps and the computed phase parameters derived from them, including phase histogram bandwidth (BW), phase standard deviation (SD), skewness, and kurtosis metrics. ${ }^{6}$ If the LV valve plane is placed too far out beyond the end of the basal myocardium, the algorithms will misinterpret random variations of background counts as contributing to the number of pixels with contractions far from the actual phase at which the majority of regional LV contractions occurred, thereby leading to the false impression of asynchronous LAD and/or RCA territories, and an inaccurate broadening of the phase histogram curve with subsequent incorrectly large values of phase $\mathrm{BW}$ and SD. As phase measurements are a relatively recent extension of MPI study computations, establishing reliable values of these is important to enable capitalizing on the added dimension this has provided when evaluating patients with CAD. ${ }^{7}$

When first presented with a newly acquired MPI dataset to be processed, users of analysis software packages frequently will first be presented with screens displaying the automated attempts to localize the LV centers and boundaries. As Malek et $\mathrm{al}^{2}$ point out, it is important to verify that these initial automated definitions are correct, or to modify them to best conform to the visual impression of optimally appropriate choices. It should be mentioned that during this initial stage of processing MPI data, it is important to also pay close attention to the overall quality of the acquired image data. Most software packages simultaneously display a cinematic playback of the original rotating data during this data processing step, if data were acquired by a rotating gamma camera, and users need to inspect this cine for issues that can affect data integrity, such as patient vertical and twisting motion. ${ }^{8}$ Even cooperative patients may be unable to control their motion, such as due to coughing or neurologic motor disorders, so that it is always important to verify the data are not 
compromised by patient motion, or to use post-acquisition motion correction software that can be used manipulate the vertical position of projection data. ${ }^{9}$ Currently, efforts are underway to enable users to correct gated SPECT MPI reconstructions simultaneously for both pulmonary and cardiac motion. ${ }^{10}$

The cine should also be inspected for possible inconsistent projection data, such as can be caused by overlying metallic devices, accidental interruption of electronic signals, and intermittent count losses due to inadequate ECG lead placement or transient arrhythmias. ${ }^{11}$ MPI studies generally are performed as gated procedures, and an important QA check prior to beginning data collection is verifying integrity of ECG leads, because if these are not placed properly then the supposed R-wave gating device will not trigger on the $\mathrm{R}$-wave but on a different portion of the trace. Also, electrical contacts must be secure or else the signal can be randomly interrupted. Relevant to these potential problems, analysis tools often are provided by manufacturers to enable users to recognize the presence of R-wave trigger gating errors during the initial data processing stage. These gating errors have the potential to affect MPI measurements, especially those related to myocardial wall thickening computations. $^{12}$

Of the issues that can cause inconsistent myocardial counts, probably the most common factors are those related to variable radiation attenuation from different projection angles. For instance, dense breast attenuation in female patients can complicate scan interpretation and influence quantified relative perfusion scores. For those SPECT systems equipped with CT attenuation correction devices, an important QA step is to verify correct registration of CT and SPECT transaxial sections, as otherwise perfusion defect artifacts can result. ${ }^{13}$

Looking to the future, developments in detector design and reconstruction algorithms have expanded the types of physiologic parameters that can be computed in Nuclear Cardiology, and these newer procedures will require additional QA steps. In recent years, several different SPECT system designs have become available that do not physically rotate around the patient, but rather collect data simultaneously from all projections over a $180^{\circ}$ arc about the patient's thorax. ${ }^{14}$ While it is possible to quantify myocardial blood flow from dynamic SPECT data acquired with a conventional rotating Ager camera, ${ }^{15}$ the advent of the newer SPECT system designs, and especially those incorporating solid state detectors for which counting efficiency is 3-5 times that of conventional Anger cameras, ${ }^{14}$ have the prospect of enabling absolute quantification of myocardial blood flow derived from dynamic SPECT acquisitions, such as with agents such as T-99m-tetrofosmin, ${ }^{16}$ in a manor analogous to the way in which rest/stress Rb-82 PET dynamic data currently are used to quantify blood flow. If that is the case, additional QA data processing procedures will be needed, including those designed to verify correct timing of bolus injections, adequacy of tracer delivery, and sufficiency of radiotracer curve clearance.

Dynamic rest/stress PET protocols are useful in evaluating CAD not only from the standpoint of quantifying blood flow, but also in that they enable computation of ejection fraction $(\mathrm{EF})$ reserve, that is, the change of EF from a resting state to a genuinely different physiologic stress state. ${ }^{17}$ Currently, this usually is performed by means of rest/stress $\mathrm{Rb}-82$ dynamic PET protocols. If it is possible, to adapt these protocols to rest/stress dynamic SPECT technology, then additional quality control procedures will need to be carried over as well, similar to those currently in use for analyzing PET data. One such protocol that recently has been reported involves low-dose/high-dose rest/regadenosine-stressTc-99m sestamibi data acquisitions using a non-rotating SPECT system. ${ }^{18}$ For instance, during the data collection stage, consistency of patient positioning (e.g., breast binding, clothing arrangement, metallic leads, arm placement, organ field of view, etc.) will be needed to facilitate comparison of rest to stress SPECT data, beyond the degree of caution currently in place for conventional SPECT protocols. In the data processing stage, verifying consistency of computed myocardial mass between stress data and rest data has been proposed as one means of assuring the validity of observed stress-induced changes in LV shape, ejection fraction, and asynchrony in $\mathrm{Rb}-82$ myocardial equilibrium gated PET data. ${ }^{19}$

In summary, the review of data processing QA procedures covered by Malik et $\mathrm{al}^{2}$ provide a good starting point for obtaining accurate quantified parameters from conventionally acquired gated SPECT MPI data. Additional attention to detail during data acquisition and additional checks of data integrity after the completion of data collection will further bolster confidence in visual and quantified findings. And, in the near future, newer equipment designs, analysis software, and novel tracers will inevitably expand the list of physiologic parameters than can be assessed in Nuclear Cardiology, the validity of which will be assured be the application of appropriate new QA checks.

\section{References}

1. Nichols K, Galt JR. Quality control for SPECT imaging. In: DePuey EG, Berman DS, Garcia EV, editors. Cardiac SPECT, vol. 39. 2nd ed. Philadelphia: Lippincott Williams \& Wilkins; 2001. pp. 17-39. 
2. Malek H, Yaghoobi N, Hedayati R. Artifacts in Quantitative analysis of myocardial perfusion SPECT, using Cedars-Sinai QPS Software. J Nucl Cardiol. 2016. doi:10.1007/s12350-016-0726-6.

3. Germano G, Kavanagh PB, Slomka PJ, Van Kriekinge SD, Pollard G, Berman DS. Quantitation in gated perfusion SPECT imaging: The Cedars-Sinai approach. J Nucl Cardiol. 2007;14:433-54.

4. Faber TL, Cooke DC, Folks RD, Vansant JP, Nichols KJ, DePuey EG, et al. Left ventricular function from gated SPECT perfusion images: An integrated method. J Nucl Med. 1999;40:650-9.

5. Ficaro EP, Lee BC, Kritzman JN, Corbett JR. Corridor4DM: The Michigan method for quantitative nuclear cardiology. J Nucl Cardiol. 2007;14:455-65.

6. Chen J, Garcia EV, Folks RD, Cooke CD, Faber TL, Tauxe EL, et al. Onset of left ventricular mechanical contraction as determined by phase analysis of ECG-gated myocardial perfusion SPECT imaging: Development of a diagnostic tool for assessment of cardiac mechanical dyssynchrony. J Nucl Cardiol. 2005;12:687-95.

7. Soman P, Chen J. Left ventricular dyssynchrony assessment using myocardial single-photon emission CT. Semin Nucl Med. 2014:44:314-9.

8. Niu X, Yang Y, Jin M, Wernick MN, King MA. Effects of motion, attenuation, and scatter corrections on gated cardiac SPECT reconstruction. Med Phys. 2011;38:6571-84.

9. Bai C, Maddahi J, Kindem J, Conwell R, Gurley M, Old R. Development and evaluation of a new fully automatic motion detection and correction technique in cardiac SPECT imaging. $\mathbf{J}$ Nucl Cardiol. 2009;16:580-9.

10. Lee T-S, Tsui BMW. The development and initial evaluation of a realistic simulated SPECT dataset with simultaneous respiratory and cardiac motion for gated myocardial perfusion SPECT. Phys Med Biol. 2015;60:1399-413.

11. Nichols K, Dorbala S, DePuey EG, Yao SS, Sharma A, Rozanski A. Influence of arrhythmias on gated SPECT myocardial perfusion and function quantification. J Nucl Med. 1999;40:924-34.
12. Nichols K, Yao SS, Kamran M, Faber TL, Cooke CD, DePuey EG. Clinical impact of arrhythmias on gated SPECT cardiac myocardial perfusion and function assessment. J Nucl Cardiol. 2001;8:1930.

13. Goetze S, Wahl RL. Prevalence of misregistration between SPECT and CT for attenuation-corrected myocardial perfusion SPECT. J Nucl Cardiol. 2007;14:200-6.

14. Slomka PJ, Patton JA, Berman DS, Germano G. Advances in technical aspects of myocardial perfusion SPECT imaging. J Nucl Cardiol. 2009;16:255-76.

15. Klein R, Hung GU, Wu TC, Huang WS, Li D, deKemp RA, et al. Feasibility and operator variability of myocardial blood flow and reserve measurements with ${ }^{99 \mathrm{~m}} \mathrm{Tc}$-sestamibi quantitative dynamic SPECT/CT imaging. J Nucl Cardiol. 2014;21:1075-88.

16. Ben Bouallegue F, Roubille F, Lattuca B, Cung TT, Macia JC, Gervasoni $\mathrm{R}$, et al. SPECT myocardial perfusion reserve in patients with multivessel coronary disease: Correlation with angiographic findings and invasive fractional flow reserve measurements. J Nucl Med. 2015;56:1712-7.

17. Van Tosh A, Votaw JR, Reichek N, Palestro CJ, Nichols KJ. The relationship between ischemia-induced left ventricular dysfunction, coronary flow reserve and coronary steal on regadenoson stress gated $82 \mathrm{Rb}$ PET myocardial perfusion imaging. J Nucl Cardiol. 2013;20:1060-8.

18. Brodov Y, Fish M, Rubeaux M, Otaki Y, Gransar H, Lemley M, et al. Quantitation of left ventricular ejection fraction reserve from early gated regadenoson stress Tc-99m high-efficiency SPECT. J Nucl Cardiol. 2016;23:1251-61.

19. Van Tosh A, Reichek N, Phippen-Nater B, Palestro CJ, Nichols KJ. Consistency of myocardial mass computations as a quality control check on rest and stress left ventricular ejection fractions computed from Rb-82 PET data. Clin Nucl Med. 2014;39:593-7. 